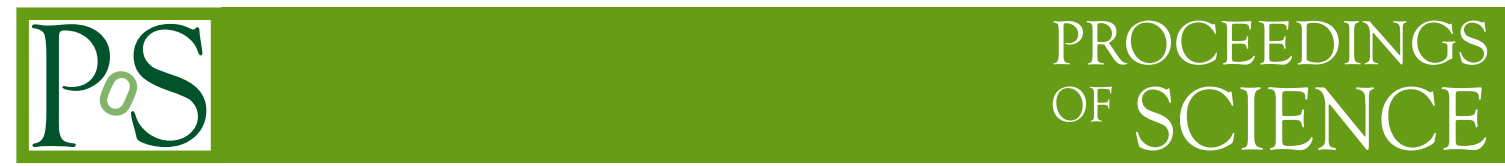

\title{
Event generators for top quark production and decays
}

\author{
Sergey Slabospitsky* \\ Institute for High Energy Physics, Protvino, Russia \\ E-mail: Sergey.Slabospitsky@cern.ch
}

This contribution provides brief descriptions the current status of the basic Monte Carlo event generators for top-quark production and decays.

International Workshop on Top Quark Physics

January 12-15, 2006

Coimbra, Portugal

${ }^{*}$ Speaker. 


\section{INTRODUCTION}

The Standard Model (SM) top quark couplings are uniquely fixed by the principle of gauge invariance, the structure of the quark generations, and a requirement of including the lowest dimension interaction Lagrangian [1]. Within the SM the top quark is considered as a point-like particle. It should be stressed, that within the SM all top-quark production properties and decays are evaluated with high accuracy without any phenomenological parameters. The total cross section production as well as the differential distributions are calculated with $\mathscr{O}(10 \%)$ accuracy [1]. The top quark decays through one decay channel, $t \rightarrow b W^{+}$(other decay channels have very small branching ratios, less then $\left.\mathscr{O}\left(10^{-3}\right)\right)$. Due to a very small life-time $\left(\tau_{t} \sim 10^{-24} \mathrm{sec}, \tau_{t} \ll 1 / \Lambda_{\mathrm{QCD}}\right)$ the top-quark decays long before it can hadronize. Therefore, it is unlikely to expect the formation top-hadrons, $T(t \bar{t})$ - or $M(t \bar{q})$-mesons and $\Lambda(t q q)$-baryons. The search for anomalous (i.e. non-SM) interactions is one of the main motivations for studying top-quark physics. In addition, very often the events with the top quarks are backgrounds to new physics that we hope to discover.

The physics of the top quark at the LHC has been studied in great detail [1], including in many cases a realistic simulation of the detectors (see talks at this Workshop). The goal of this presentation is to give a short review of Monte Carlo (MC) generators that provide a simulation of the processes with top-quark production and decays (the detailed considerations could be found elsewhere [1, 凤

\section{GENERAL SCHEME OF GENERATOR}

A general scheme of full event simulation assumes the evaluation of the hard process (the cross section value, the incoming and outgoing particle's momenta and colours), then to evolve the event through a parton showering and hadronization the coloured products of shower, and the decay of the unstable particles. As a result an event information contains the momenta of the final hadrons, leptons and photons and positions of their decay vertexes. Typically such information contains also the characteristics (momenta, colours, $\mathrm{KF}$-codes, mother's and daughter's relations) of all intermediate partons (quarks, gluons, gauge bosons, unstable physical particles, etc) that provide to trace-back the history of particles production insight of event. Any such generator by using an acceptance-rejection method (Von Neumann) returns weighted event. The most popular full event generators, like HERWIG [3], PYTHIA [4], ISAJET [5], and SHERPA [6] include all such steps of simulation.

We start with the consideration of the specific moments related to the processes with topquarks and describe their realization in MC generators. The diagrams describing top-pair and single top production (at leading order - LO) are presented on Figs 1, 2.
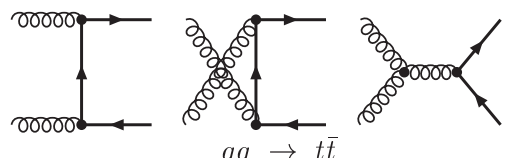

Figure 1: $t \bar{t}$ production (QCD)



$q \bar{q} \rightarrow t \bar{t}$ 



Figure 2: single top production (electro-weak)

In almost all cases one needs to know an information not only about final top-quark, but also about it decay products. Therefore, it is appeared a question on "how to include top decays ?". The natural and correct way is to calculate an exact amplitude including the initial partons and final state particles. However, the full amplitude typically consists many diagrams without intermediate top-quarks. For examples, the full amplitude of the process $g g \rightarrow t \bar{t}$ with the both final $W$-bosons decaying into electrons has many other diagrams without $t$-quarks. In particular, there is at least one diagram with two Z-bosons: $g g \rightarrow b \bar{b} Z Z$ with one $Z$ decaying into $e^{+} e^{-}$and second $Z \rightarrow v_{e} \bar{v}_{e}$. Typically, all such non-resonant diagrams (i.e. without top-quark) will give a very small contribution to "resonance" region, where when the invariant masses of the final particles are very close to $t$-quark and $W$-boson masses (e.g. $M\left(b e^{ \pm} v_{e}\right) \approx m_{t}$ and $\left.M\left(e^{ \pm} v_{e}\right) \approx M_{W}\right)$. Therefore, for this case it is useful to explore a Narrow Width Approximation (NWA). It means that one take into account only diagrams with intermediate top quarks and consider all $t$-quarks as "on-shell" particles:

$$
\frac{1}{\left(p_{t}^{2}-m_{t}^{2}\right)^{2}+m_{t}^{2} \Gamma_{t}^{2}}=\frac{\pi}{m_{t} \Gamma_{t}} \delta\left(p_{t}^{2}-m_{t}^{2}\right)
$$

As a result one can factorize the production and decays of the top quark. After that, typically there are appeared three questions: how to include top decays ?; how to reproduce Breit-Wigner resonance shape ?; how to include top spin (polarization)?

\section{TOP DECAYS}

\subsection{Decays}

Within the SM in $99.9 \%$ of case the top-quark decays into $b W$ final state: $t \rightarrow b W, W \rightarrow f \bar{f}^{\prime}$. The matrix element describing this decay is well known [1]:

$$
\left|M\left(t \rightarrow b f \bar{f}^{\prime}\right)\right|^{2} \propto \frac{\left(p_{b} p_{f}\right)\left(p_{t} p_{\bar{f}^{\prime}}\right)}{\left(p_{W}^{2}-M_{W}^{2}\right)^{2}+\Gamma_{W}^{2} M_{W}^{2}}
$$

In fact, this is only one decay channel that included in almost all generators. Other interesting SM channels (e.g $t \rightarrow b W^{*} Z^{*} \rightarrow b f \bar{f}^{\prime} \ell^{+} \ell^{-}$) are still not available at generator level. At the same time few other decay channels (due to non-SM physics) are included in generators. In particular, they are:

$\diamond$ SUSY: $t \rightarrow b H^{+}$(almost all packages)

$\diamond$ FCNC: $t \rightarrow q g, q Z, q \gamma($ TopReX [20])

$\diamond t \rightarrow b W\left(\rightarrow f \bar{f}^{\prime}\right)$ with anomalous interactions ( $V+A$ and tensor couplings, ONETOP [25]) 


\subsection{The Breit-Wigner resonance shape}

The usage of Narrow Width Approximation (NWA) assumes that all top quarks in any event have the same default mass $\left(m_{t}\right)$. There are two approaches, that provide to reproduce the BreitWigner resonance shape due to the finite decay width of the top-quark.

With "smearing-mass" method the matrix elements are calculated for default $m_{t}$ mass [屯] . Then for each event Breit-Wigner distribution

$$
f\left(\tilde{m}_{t}\right) \propto \frac{1}{\left(\tilde{m}_{t}^{2}-m_{t}^{2}\right)^{2}+\Gamma_{t}^{2} m_{t}^{2}}
$$

is used to generate separate masses $\left(\tilde{m}_{t}\right)$ for top-quarks. Then, the momenta and energies of all final-state particles should re-evaluated. For example, for $t \bar{t}$ pair production with new $\tilde{m}_{1}(t)$ and $\tilde{m}_{2}(\bar{t})$ masses one has:

$$
t \bar{t}: E_{1}^{*}(t)=E_{2}^{*}(\bar{t})=\sqrt{\hat{s}} / 2 \Longrightarrow \tilde{E}_{1 / 2}=\frac{\sqrt{\hat{s}} \pm \tilde{m}_{1}^{2}(t) \mp \tilde{m}_{2}^{2}(\bar{t})}{2 \sqrt{\hat{s}}}
$$

This method certainly is not unique, but normally provide a sensible behavior. It can be applied for event with any number of top quarks in final state $(t, t \bar{t}, \ldots)$.

Another proposed method (modified NWA [25]) assumes the generation of new $\tilde{m}_{t}$ for $t$-quark in the event by using of Breit-Wigner distribution. Then, the squared matrix element $\left(|M|^{2}\right)$ is calculated with this new $\tilde{m}_{t}$. Note, that such trick can be used only for the process with one top quark in the final state (i.e. not for $t \bar{t}$ production).

\subsection{How to include top polarization}

Since the top quark decays before hadronization, its spin properties are not spoiled. Therefore spin correlations in top production and decays is an interesting issue in top-quark physics. The simple way to include top polarization assumes usage of the helicity amplitude technique [7]. An equivalent (and sometimes more simple for practical usage) method [8] is realized in TAUOLA [9] package. In this method the matrix element squared $|M|^{2}$ can be represented in the "factorized" form.

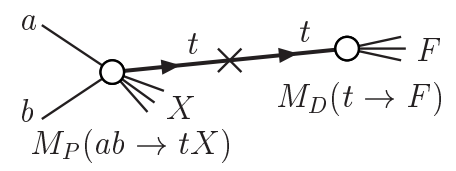

Figure 3: an equivalent method (TAUOLA)

For the process with one final top-quark (see Fig. 3) the full amplitude squared has the form:

$$
\begin{aligned}
& |M(a b \rightarrow F+X)|^{2}=\frac{\pi}{\Gamma_{t} m_{t}} \delta\left(p_{t}^{2}-m_{t}^{2}\right) \times\left|M_{P}^{0}(a b \rightarrow t+X)\right|^{2}\left(1+v_{i} h_{i}\right)\left|M_{D}^{0}(t \rightarrow F)\right|^{2} \\
& \left|M_{P}(a b \rightarrow t+X)\right|^{2}=\left|M_{P}^{0}(a b \rightarrow t+X)\right|^{2}(1+(v s)) \\
& \left|M_{D}(t \rightarrow F)\right|^{2}=\left|M_{D}^{0}(t \rightarrow F)\right|^{2}(1+(h s))
\end{aligned}
$$


where the amplitudes $\left|M^{0}\right|^{2}$ describe the production and decay of unpolarized top-quark, $s$ is the $t$-quark spin $\left(\left(p_{t} s\right)=0\right)$, and $v^{\mu}, h^{\mu}$ are so-called "polarization" vectors [8].

For example, the spin-dependent matrix element squared describing top-quark decay $t \rightarrow b \ell^{+} v$ can be re-presented in the form as follows:

$$
|M|^{2} \propto \frac{\left(p_{b} p_{v}\right)\left(p_{t} p_{\ell}\right)}{\left(p_{W}^{2}-M_{W}^{2}\right)^{2}+\Gamma_{W}^{2} M_{W}^{2}}\left(1-\frac{m_{t}\left(p_{\ell} s\right)}{\left(p_{t} p_{\ell}\right)}\right) \Rightarrow v^{\mu}=-\frac{m_{t} p_{\ell}^{\mu}}{\left(p_{t} p_{\ell}\right)} \Rightarrow-\vec{n}_{\ell}^{*}
$$

where $\vec{n}_{\ell}^{*}$ is the direction of $\ell^{+}$momentum in $t$-quark rest frame.

\section{MATCHING ALGORITHMS}

\subsection{Matching Parton Showers and Matrix Elements}

Recently a substantial progress has been achieved in simulation of the process with multi-jet final states [10, 12]. Indeed, the description of multi-jets obtained from the shower evolution is inaccurate, since hard radiation at large angle is suppressed by the angular ordering prescription. The available generators (ALPGEN, CompHEP, Madevent, etc) provide a generation of top production with up to 6-jets [11, 13, 14, 15]. Due to the complexity of the matrix elements(ME) evaluation for these many-body configurations, calculation are normally available only for LO cross sections.

An approach in which multi-parton events generated using the exact leading order ME generator can be consistently evolved into multi-jet final states by means of a shower MC. The main problem is how to eliminate the double counting, where jets can arise from both the higher-order calculation and from the hard emission during the shower evolution.

There are several approaches to this problem, aiming at different levels of accuracy. The first one ("matrix-element correction" technique [16]) corrects the approximate ME for the emission of the hardest gluon in a given process by using the exact LO ME. The second is known as CKKW [17]; its goal is to implement multi-jet ME corrections at the leading, or next-to-leading logarithmic level. This method assumes a separation of the multi-jet phase-space into the domains covered by the ME calculation and the domains covered by the shower evolution. Then by means of Sudakov re-weighting the ME weight to reproduce the probability of an exclusive $\mathrm{N}$-jet final state from the inclusive parton-level $\mathrm{N}$-jet rate. This allows to add parton-level event samples of different jet multiplicity.

An additional prescription is proposed in [11] ("MLM prescription"). In this approach the partons from the ME calculation are matched to the jets reconstructed after the perturbative shower. Parton-level events are defined by a minimum $E_{T}$ threshold $E_{T}^{m i n}$ for the partons, and a minimum separation among them, $\Delta R_{j j}>R_{\min }$. However, no Sudakov re-weighting is applied. Rather, events are showered, without any hard-emission veto during the shower. After evolution, a jet cone algorithm with cone size $R_{\min }$ and minimum transverse energy $E_{T}^{\min }$ is applied to the final state. Starting from the hardest parton, the jet which is closest to it in $(\eta, \phi)$ is selected. If the distance between the parton and the jet centroid is smaller than $R_{\min }$, the parton and the jet match. The matched jet is removed from the list of jets, and matching for subsequent partons is performed. The event is fully matched if each parton has a matched jet. Events which do not match are rejected. For events which satisfy matching, it is furthermore required that no extra jet, in addition to those 
matching the partons, be present. Events with extra jets are rejected, a suppression replacing the Sudakov re-weighting used in the CKKW approach. Events obtained by applying this procedure to the parton level with increasing multiplicity can then be combined to obtain fully inclusive samples spanning a large multiplicity range. Events with extra jets are not rejected in the case of the sample with highest partonic multiplicity. Fig. $\theta$ presents an illustration of above-described examples [11, 12].
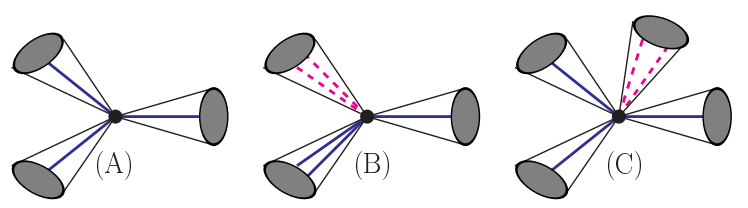

Figure 4: Few examples of matching. The solid line corresponds to parton from a hard process, while the dashed one presents a parton emitted by the shower. (A) $N_{\text {jet }}=N_{\text {part }}=3$, the event is matched and kept. (B) $N_{\text {jet }}=N_{\text {part }}=3$, but $N_{\text {matched }}=2$, the event should be rejected. (C) $N_{\text {matched }}=N_{\text {part }}=3$, but $N_{\text {jet }}=4$, the event is matched and kept for inclusive sample, but should be rejected for exclusive samples.

\subsection{Matching with and additional $b$-quark}

Very simple procedure is proposed for the process of $t$-channel single-top production [19], where one has two complementary subprocess (see Fig. 5):

$$
\text { " } 2 \rightarrow 2^{\prime \prime} q b \rightarrow q^{\prime} t \quad \text { and } \quad \text { " } 2 \rightarrow 3^{\prime \prime} q g \rightarrow q^{\prime} t \bar{b}
$$

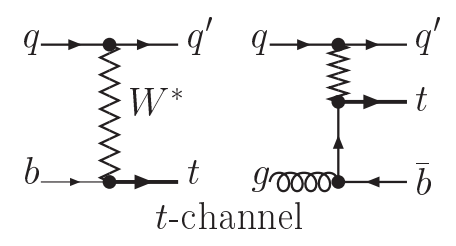

Figure 5: Two subprocess of the single top production.

This matching procedure is based on the consideration of the transverse momentum $\left(p_{\top}(\bar{b})\right)$ of additional $\bar{b}$-quark [19].

The matching procedure starts from simulation of the events with $t$-quark production due to $2 \rightarrow 2$ process. Such an event has an additional $\bar{b}$-quark generated by MC showering generator (i.e. PYTHIA or HERWIG). Any event from this sample is accepted if the transverse momentum of the additional $\bar{b}$-quark is smaller than some value $p_{0}$. Second sample consists of events from $2 \rightarrow 3$ process. The event from this sample is accepted if the additional $\bar{b}$-quark from a hard process has $p_{\top}(\bar{b})>p_{0}$. Thus, the resulting (total) sample of $t$-quark production events is the sum of two contributions:

$$
\begin{aligned}
N(p p \rightarrow t X)_{t-\text { channel }} & =N^{(2 \rightarrow 2)}\left(p p \rightarrow t q^{\prime} ; p_{\top}(\bar{b})<p_{0}\right) \\
& +N^{(2 \rightarrow 3)}\left(p p \rightarrow t q^{\prime} \bar{b} ; p_{\top}(\bar{b}) \geq p_{0}\right)
\end{aligned}
$$


The corresponding $p_{\top}(\bar{b})$-distributions are shown in Fig. 6 .

This method of generation of $t$-channel single-top production is realized in SingleTop [19] and TopReX [20] codes.

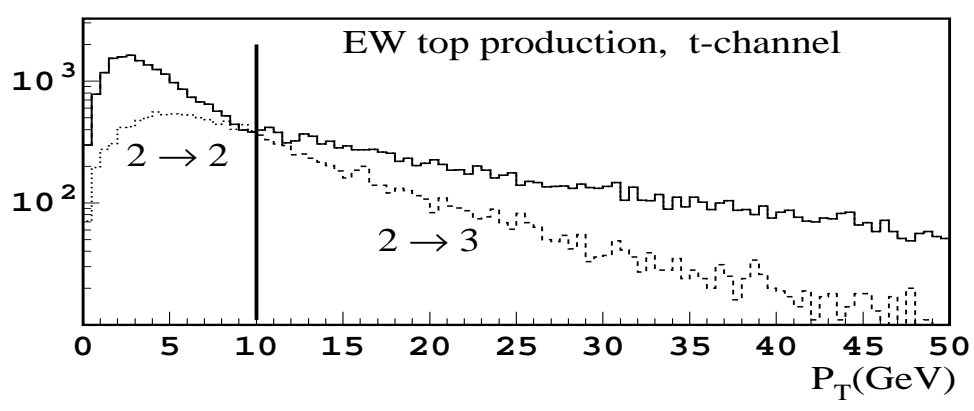

Figure 6: $p_{\top}(\bar{b})$ distributions of the additional $\bar{b}$-quark production in " $2 \rightarrow 2$ " (the dotted curve) and " $2 \rightarrow 3$ " (the dashed curve) processes. The solid histogram is the sum of these two contributions. The vertical line corresponds to parameter value $p_{0}=10 \mathrm{GeV}$.

\section{NLO CORRECTIONS}

Recently a substantial progress has been achieved in the calculations of the single-top production at NLO [24, 25, 26, 27]. The radiation effects are included in the initial and final states, as well as into decays. These generators (like ZTOP [24], ONETOP [25] and the code based on MCFM generator [26]) provide a generation of total cross section and differential distributions.

One really needs to include NLO corrections in ME generators. Indeed, only shower MC's provide a representation of the final state complete enough to allow realistic detector simulations. On the other hand, the inclusion of NLO effects in fixed-order ME MC's leads to distributions which are not positive definite. The naive introduction of NLO matrix element could lead to double counting, since as shower MC generators already incorporate part of the NLO effects (a real emissions, as well as virtual effects included in the Sudakov form factors). This problem was successfully solved (see the article [18], where it was shown how this merging can be done very effectively). This method is implemented in MC@NLO generator [23] describing heavy-quark pair, Higgs, DY and gauge boson pair production.

The inclusion of NLO corrections in the shower MC guarantees that total cross-sections generated by the MC reproduce those of the NLO ME calculation, thereby properly including the $K$ factors and reducing the systematic uncertainties induced by renormalization and factorization scale variations. At the same time, however, the presence of the higher-order corrections generated by the shower will improve the description of the NLO distributions, leading to departures from the parton-level NLO result. This is shown, for example, in Fig. 7. which shows the $p_{T}$ spectrum of a $t \bar{t}$ pair resulting from the pure NLO calculation, from the LO shower, and from the MC@NLO improvement. At large $p_{T}$, a region dominated by the NLO effects, MC@NLO faithfully reproduces the hard, large-angle emission distribution given by the NLO matrix elements. At small $p_{T}$, a region dominated by multiple radiation and higher-order effects, the MC@NLO departs signifi- 
cantly from the NLO result, while properly incorporating the Sudakov re-summation effects only available via the IS shower evolution.



Figure 7: Transverse momentum distribution of top quark pairs using three different approaches: the LO HERWIG MC, the parton-level NLO MC, and the merging of the two into MC@ NLO. Figure from [22].

Recently, NLO corrections to the single-top production processes ( $t$ - and $s$-channels) are included at MC@NLO event generator [27]. The comparison MC@NLO and HERWIG results for the single top production are given on Fig. 8. Note, that the both generators give very close results for the highest $p_{T}$ jet distribution (the left histogram on Fig. 8), while MC@ NLO predicts much harder $p_{T}$-spectrum for $b$-jet (not from top decay).
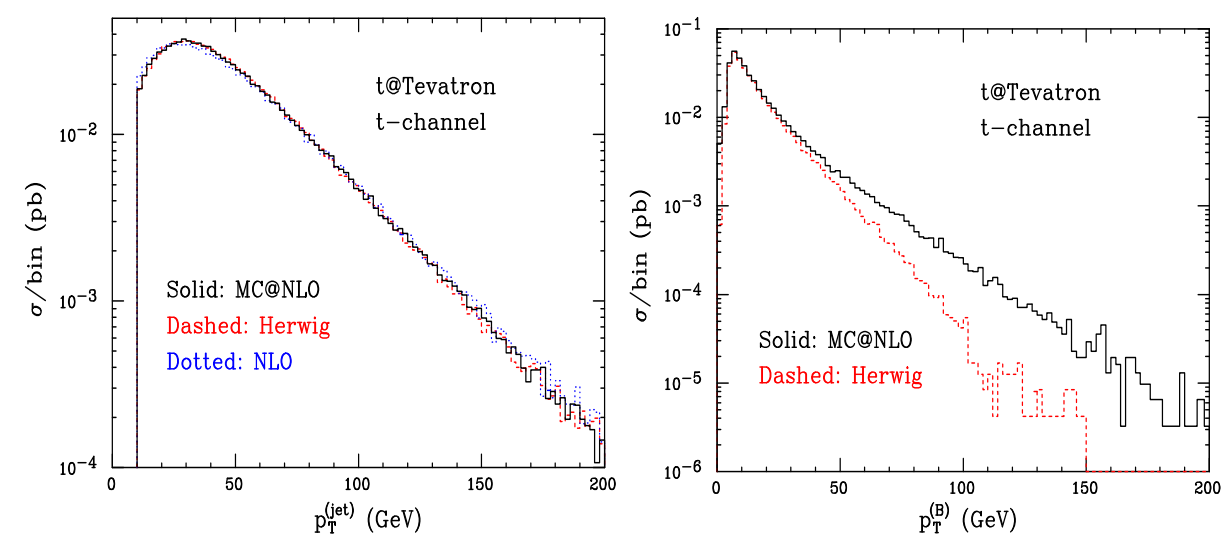

Figure 8: The transverse momentum distributions for the highest $p_{T}$ jet and $b$-jet (not from top decay).

\section{EVENT GENERATORS}

This section presents brief description of the generators. Only few basic comments and the list of included top-quark production process are given for each entry.

\subsection{Full event simulation packages}

These packages provide a full event simulation including the hard process generation, showering and hadronization with subsequent decays of the unstable hadrons. 


\section{•HERWIG [B]}

contains a wide range of Standard Model, Higgs and supersymmetric processes. It uses the partonshower approach for initial- and final-state QCD radiation, including colour coherence effects and azimuthal correlations both within and between the jets. HERWIG is particularly sophisticated in its treatment of the subsequent decay of unstable resonances, including full spin correlations for most processes.

Processes included: $t \bar{t}$, single top ( $t, s$ channels), $t \bar{t} H, Z t \bar{t}, g b \rightarrow t H^{+}$

-PYTHIA [曲

is a general-purpose generator for hadronic events in $\mathrm{pp}, \mathrm{e}^{+} \mathrm{e}^{-}$and $e p$ colliders. It contains a subprocess library and generation machinery, initial- and final-state parton showers, underlying event, hadronization and decays, and analysis tools.

Processes included: $t \bar{t}$, single-top $\left(t, s\right.$ channels), $t \bar{t} H, g b \rightarrow t H^{+}$, no spin correlations

-ISAJET [5]

is a general-purpose generator for hadronic events. ISAJET is based on perturbative QCD plus phenomenological models for parton and beam jet fragmentation.

Processes included: $t \bar{t}$, no spin correlations

-SHERPA [6]

is a new multi purpose event generator with a powerful matrix element generator AMEGIC++

\subsection{Tree level matrix element generators}

Such packages generate the hard processes kinematic quantities, such as masses and momenta, the spin, the colour connection, and the flavour of initial- and final-state partons. Then such information is stored in the "Les Houches" format [29] and is passed to full event simulation generator (like PYTHIA or HERWIG).

\section{-ALPGEN [11]}

is designed for the generation of the SM processes in hadronic collisions, with emphasis on final states with large jet multiplicities. It is based on the exact LO evaluation of partonic matrix elements, as well as top and gauge boson decays with helicity correlations. The code generates events in both a weighted and unweighted mode.

Processes included: $t \bar{t}+$ up to 6jets, single top: $t q, t b, t W, t b W$ (no extra jets), $t \bar{t} t \bar{t}+$ up to 4 jets, $t \bar{t} b \bar{b}+$ up to 4 jets, $H t \bar{t}+$ up to 4 jets, $W / Z t \bar{t}+$ up to 4 jets

\section{-CompHEP [13]}

CompHEP computes squared Feynman diagrams symbolically and then numerically calculates cross sections and distributions. The output event's information in the "Les Houches" format [29].

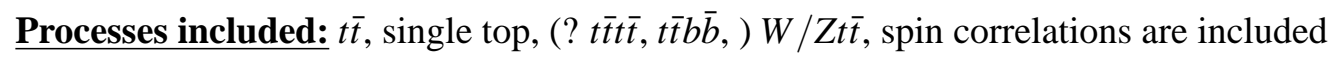

-MadEvent [14]

is a multi-purpose, tree-level event generator, which is powered by the matrix element generator MadGraph [15]. MadGraph automatically generates the amplitudes for all the relevant subprocesses and produces the mappings for the integration over the phase space.

Processes included: $t \bar{t}+$ up to 3 jets, single top, $t \bar{t} b \bar{b}+$ up to 1 jet, $H t \bar{t}$ up to 2 jets 
-MC@NLO [23] combines a Monte Carlo event generator with exact NLO calculations of rates for QCD processes at hadron colliders.

Processes included: $t \bar{t}$, single top ( $t$ - and $s$-channel)

-AcerMC [21] (see talk of B.Krersevan in this conference).

AcerMC is dedicated for generation of the Standard Model background processes in $p p$ collisions at the LHC

Processes included: $t \bar{t}$, single top, $t \bar{t} t \bar{t}, t \bar{t} b \bar{b}, W / Z t \bar{t}$, spin correlations are included

-SingleTop [19] generator is based on the CompHEP [13] package.

processes: $t$-channel single top production $(2 \rightarrow 2+2 \rightarrow 3$, spin correlations are included

-TopReX [20]

provides a simulation of several important processes in hadronic collision, not implemented in PYTHIA. Several top-quark decays channels are included: the SM channel $\left(t \rightarrow q W^{+}, q=d, s, b\right), b$ quark and charged Higgs $\left(t \rightarrow b H^{+}\right)$and the channels with flavor changing neutral current (FCNC): $t \rightarrow u(c) V, V=g, \gamma, Z$. The implemented matrix elements take into account spin polarizations of the top quark.

Processes included: $g g(q \bar{q}) \rightarrow t \bar{t}$, single-top production $\left(t-, s\right.$, and $t W$-channel), $q \bar{q}^{\prime} \rightarrow H^{ \pm *} \rightarrow t \bar{b}$, $q \bar{q} \rightarrow W^{*} / Z^{*} Q \bar{Q}$ with $W^{*} / Z^{*} \rightarrow f \bar{f}$ and $Q=c, b, t, g u(c) \rightarrow t \rightarrow b W$ (due to FCNC).

-MCFM [28] included the matrix elements at next-to-leading order and incorporate full spin correlations.

Processes included: $t \bar{t}$, single top ( $t$ - and $s$-channel), $H t \bar{t}, W / Z t \bar{t}$

$\bullet$ ZTOP [24] code includes the full NLO-corrections to single top production ( $t$ - and $s$-channel).

-ONETOP [25] code include full NLO-corrections to single top production ( $t$ - and $s$-channel) and top-quark decay.

\section{CONCLUSIONS}

Recently a substantial progress had been achieved in implementation of new ideas of top events simulation and developing of event generators for the top quark production and decays.

In particular, several new processes describing the top-quark production and decays are included in the generators provided full simulation of event. Many event generators provide generation of the top-quark processes with spin correlations. Tree level generators make possible a generation of the top production processes with additional multi-jets in the final states. The codes with full NLO corrections to single-top production processes are available. Few generators include the processes with the top-quark production and decays due to interactions beyond SM.

\section{Acknowledgments}

I would like to thank the organizers of "Top 2006" for the invitation and extremely pleasant atmosphere and fruitful discussion during the conference. 


\section{References}

[1] M. Beneke et al., Top quark physics, [arXiv: hep-ph/0003033], in Standard model physics (and more) at the LHC G. Altarelli and M.L. Mangano eds., Geneva, Switzerland: CERN (2000) 529 p.

[2] M. Dobbs et al. Les Houches Guidebook to Monte Carlo Generators for Hadron Collider Physics, hep-ph/0403045

[3] G. Corcella et al., HERWIG 6: An event generator for hadron emission reactions with interfering gluons (including supersymmetric processes), JHEP 0101 (2001) 010 [arXiv: hep-ph/0011363].

Webpage: http://hepwww.rl.ac.uk/theory/seymour/herwig

[4] T. Sjöstrand et al., High-energy-physics event generation with PYTHIA 6.1, Comput. Phys. Commun. 135 (2001) 238 [arXiv: hep-ph/0010017].

Webpage: http://www.thep.lu.se/ torbjorn/Pythia.html

[5] F.E. Paige, S.D. Protopopescu, H. Baer and X. Tata, ISAJET 7.69: A Monte Carlo event generator for $p p, \bar{p} p$, and $e^{+} e^{-}$reactions, arXiv: hep-ph/0312045.

Webpage: http://www.phy.bnl.gov/ isajet/

[6] T. Gleisberg, S. Hoeche, F. Krauss, A. Schalicke, S. Schumann and J. Winter, Event generator for the LHC, arXiv:hep-ph/0508315.

Webpage: http://www.physik.tu-dresden.de/ krauss/hep/

[7] P. Richardson, Spin correlations in Monte Carlo simulations, JHEP 0111 (2001) 029 [arXiv: hep-ph/0110108].

[8] S. Jadach and Z. Was, QED $\mathscr{O}\left(\alpha^{3}\right)$ Radiative Corrections To The Reaction $e^{=} e^{-} \rightarrow \tau^{+} \tau^{-}$Including Spin And Mass Effects. (Erratum), Acta Phys. Polon. B15 (1984) 1151 [Erratum-ibid. B16 (1985) 483].

[9] S. Jadach, J.H. Kuhn and Z. Was, Tauola: A Library Of Monte Carlo Programs To Simulate Decays Of Polarized Tau Leptons, Comput. Phys. Commun. 64 (1990) 275.

Webpage: http://wasm.home.cern.ch/wasm/goodies.html

[10] M.L. Mangano, QCD tools for the LHC, eConf C030614 (2003) 015 [arXiv: hep-ph/0312117].

[11] M.L. Mangano, M. Moretti, F. Piccinini, R. Pittau, and A.D. Polosa, ALPGEN, a generator for hard multiparton processes in hadronic collisions, JHEP 0307 (2003) 001 [arXiv: hep-ph/02 06293]. Webpage: http://mlm.home.cern.ch/mlm/alpgen/

[12] S. Hoche, F. Krauss, N. Lavesson, L. Lonnblad, M. Mangano, A. Schalicke, and S. Schumann, Matching parton showers and matrix elements, arXiv : hep-ph/0602031.

[13] E. Boos, V. Bunichev, M. Dubinin, L. Dudko, V. Edneral, V. Ilyin, A. Kryukov, V. Savrin, A. Semenov, A. Sherstnev (the CompHEP collaboration) Webpage: http://theory.sinp.msu.ru/comphep

[14] F. Maltoni and T. Stelzer, MadEvent: Automatic event generation with MadGraph, JHEP 0302 (2003) 027 [arXiv: hep-ph/0208156].

Webpage: http://madgraph.physics.uiuc.edu

[15] T. Stelzer and W. F. Long, Automatic generation of tree level helicity amplitudes, Comput. Phys. Commun. 81 (1994) 357 [arXiv: hep-ph/9401258]. 
[16] M.H. Seymour, Matrix element corrections to parton shower algorithms, Comput. Phys. Commun. 90 (1995) 95 [hep-ph/9410414].

[17] S. Catani, F. Krauss, R. Kuhn and B.R. Webber, QCD matrix elements + parton showers, JHEP 0111 (2001) 063 [arXiv: hep-ph/0109231];

L. Lonnblad, Correcting the colour-dipole cascade model with fixed order matrix elements, JHEP 0205 (2002) 046 [arXiv: hep-ph/0112284].

[18] S. Frixione and B. R. Webber, Matching NLO QCD computations and parton shower simulations, hep-ph/0204244;

S. Frixione, P. Nason and B.R. Webber, Matching NLO QCD and parton showers in heavy flavour production, JHEP 0308 (2003) 007 [hep-ph / 0305252 ].

[19] E. Boos, L. Dudko, V. Savrin, CMS Note 2000/065 (2000).

[20] S.R. Slabospitsky and L. Sonnenschein, TopReX generator (version 3.25): Short manual, Comput. Phys. Commun. 148 (2002) 87 (2002) [arXiv: hep-ph/0201292].

Webpage: http://cmsdoc.cern.ch/ spitsky/toprex/toprex.html

[21] B.P. Kersevan and E. Richter-Was, The Monte Carlo event generator AcerMC version 2.0 with interfaces to PYTHIA 6.2 and HERWIG 6.5, arXiv : hep-ph / 0405247 ;

B.P. Kersevan and E. Richter-Was, The Monte Carlo event generator AcerMC version 1.0 with interfaces to PYTHIA 6.2 and HERWIG 6.3, Comput. Phys. Commun. 149 (2003) 142 [arXiv: hep-ph/0201302].

Webpage: http: //borut.home.cern.ch/borut/

[22] S. Frixione, P. Nason and B. R. Webber, Matching NLO QCD and parton showers in heavy flavour production, JHEP 0308 (2003) 007 [hep-ph / 0305252 ].

[23] S. Frixione and B.R. Webber, The MC@NLO 3.2 event generator, arXiv: hep-ph/0601192. Webpage: http: / /www.hep.phy.cam.ac.uk/theory/webber/MCatNLO

[24] B.W. Harris, E. Laenen, L. Phaf, Z. Sullivan and S. Weinzierl, The fully differential single top quark cross section in next-to-leading order QCD, Phys. Rev. D66 (2002) 054024 [arXiv: hep-ph/0207055];

Z. Sullivan, Understanding single-top-quark production and jets at hadron colliders, Phys. Rev. D70 (2004) 114012 [arXiv: hep-ph/0408049].

[25] Q.H. Cao, R. Schwienhorst, J.A. Benitez, R. Brock, and C.-P. Yuan, Next-to-leading order corrections to single top quark production and decay at the Tevatron. II: t-channel process, Phys. Rev. D72 (2005) 094027 [arXiv: hep-ph/0504230];

Q.H. Cao, R. Schwienhorst and C.-P. Yuan, Next-to-leading order corrections to single top quark production and decay at Tevatron. I: s-channel process, Phys. Rev. D71 (2005) 054023 [arXiv: hep-ph/0409040];

Q.H. Cao and C.-P. Yuan, Single top quark production and decay at next-to-leading order in hadron collision, Phys. Rev. D71 (2005) 054022 [arXiv: hep-ph/ 0408180 ].

[26] J. Campbell, R.K. Ellis and F. Tramontano, Single top production and decay at next-to-leading order, Phys. Rev. D70 (2004) 094012 [arXiv : hep-ph/ 0408158 ].

[27] S. Frixione, E. Laenen, P. Motylinski and B.R. Webber, Single-top production in MC@NLO, arXiv:hep-ph/0512250.

[28] J. Campbell and K. Ellis, MCFM - Monte Carlo for FeMtobarn processes, Webpage: http://mcfm.fnal.gov/

[29] E. Boos et al., Generic user process interface for event generators, arXiv : hep-ph/0109068. 